\title{
Clear cell sarcoma-A review
}

\author{
Rami Mossad Ibrahim ${ }^{\mathrm{a}, *}$, Signe Steenstrup Jensen ${ }^{\mathrm{b}}$, Jacob Juel ${ }^{\mathrm{c}}$ \\ a Department Plastic and Reconstructive Surgery, Herlev Hospital, Copenhagen, Denmark \\ ${ }^{\mathrm{b}}$ Department of Plastic and Reconstructive Surgery, Odense University Hospital, Odense, Denmark \\ ${ }^{\mathrm{c}}$ Department of Plastic and Reconstructive Surgery, Aalborg University Hospital, Aalborg, Denmark
}

\section{A R T I C L E I N F O}

\section{Keywords:}

Sarcoma

Clear cell sarcoma

Malignant melanoma of the soft tissue

Treatment of clear cell sarcoma

\begin{abstract}
A B S T R A C T
Clear cell sarcoma (CCS) previously known as malignant melanoma (MM) of the soft tissue, although, similar in morphology to MM, contemporary histopathologic and cytogenetic techniques have made this diagnosis obsolete, as it is now possible to distinguish between CCS and MM. CCS is often diagnosed in young adults with median age of 25 years. Overall mortality is generally poor, and the 5-year survival is between 40 and $60 \%$. Hence, early diagnosis and radical surgery are key in the treatment of this extremely rare malignancy of the soft tissue comprising only about $1 \%$ of all sarcomas. This article present an overview of this rare malignancy.
\end{abstract}

\section{Introduction}

Clear cell sarcoma (CCS) is an extremely rare and aggressive subtype of sarcoma with melanocytic differentiation both immunohistochemically, ultrastructural and genomic. ${ }^{1,2}$ It comprises approximately $1 \%$ of all diagnosed sarcomas. ${ }^{3}$ Furthermore, the prognosis is poor due to predilection for metastasis at an early stage to the lymphatic system and the lungs. ${ }^{4}$ CCS is thought to derive from the neural crest cells. ${ }^{5}$ As a whole, sarcomas arises from the mesenchymal tissues, such as bone, muscle, fat, connective tissue, blood vessels and nerves. ${ }^{5}$ Hence, corresponding tissue origin of clear cell sarcoma, which was first described by Enzinger et al in 1965. ${ }^{6}$ The name CCS originates from the initial histopathological findings of clear cells, as the cytoplasm of the tumor cells may appear clear. ${ }^{6}$ The term malignant melanoma of the soft tissue was previously used, however, contemporary histopathologic and cytogenetic techniques have made this diagnosis obsolete, as pathologist are able to distinguish between CCS and malignant melanoma today. ${ }^{7}$ In $95 \%$ of the cases, the CCS arises in the lower extremities, particularly the foot and ankle. It presents as an indolent, growing and painless mass situated in the deep soft tissue or beneath the fascia. ${ }^{4,8-10}$ CCS occur at all ages, but is most often observed in the third decade. ${ }^{11-14}$ The mean age at the time of diagnosis is approximately 25 years and CCS is more common in Caucasians than in African Americans or Asians, without any gender predilection. ${ }^{11-14}$ The aetiology of CCS is virtually unknown, and the exact cause and the mechanisms of formation remains to be elucidated. Genetic defects occurring on account of certain chromosomal exchanges is thought to play a key role in the formation. Hence, CCS is repeatedly seen in relation to specific genetic transformations. Only a few known risk factors are described e.g. exposure to chemicals (vinyl chloride, arsenic), chronic tissue irritation (lymphedema, foreign body implants) and radiation. ${ }^{5}$

\section{Diagnosis}

Compared to the aforementioned, identifying and diagnosing CCS is challenging. Quick and accurate diagnosis is dependent on the level of experience of the doctor at the initial examination and fast referral to specialized units with relevant experience, such as tertiary sarcoma centres are imperative. The mean time from onset of symptoms to confirmation of the diagnosis is often several months, which could be the result of an initial suspicion of a benign tumor. ${ }^{13,14}$ This prolonged time to relevant examinations and to diagnosis may lead to a more advanced disease at the time of diagnosis and therefore a more sinister prognosis. ${ }^{5,14,15}$

\section{Radiological examination}

Hitherto, the most relevant initial investigation in the diagnosis of CCS is magnetic resonance imaging (MRI). Currently, no exact radiological criteria to differentiate between malignant and benign tumors in the soft tissue exist. ${ }^{7,16-18}$ However, today a combination of different imaging tools is used to provide a tentative diagnosis, while the final diagnosis of CCS is made, based on the resulting or concomitant pathological examination.

MRI is the leading and most established modality to identify the primary tumor and its relations to adjacent structures. ${ }^{17}$ In addition,

\footnotetext{
* Corresponding author. Bredgade 58a, 4. sal, DK-1260, København K, Denmark.

E-mail addresses: mossadjr@hotmail.com (R.M. Ibrahim), signe330@gmail.com (S. Steenstrup Jensen), Jacob.juel@rn.dk (J. Juel).
} 


\section{Horizontal section with contrast}

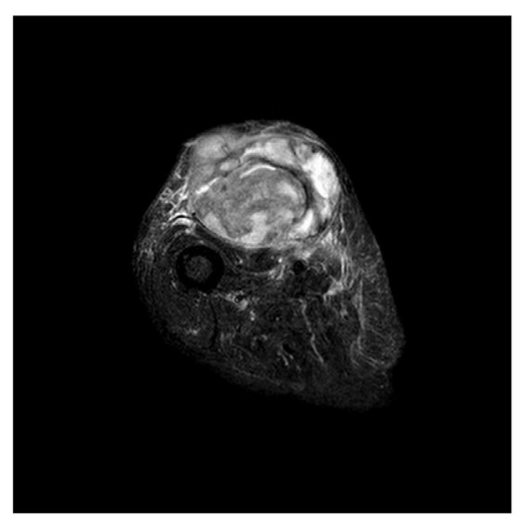

Horizontal section without contrast

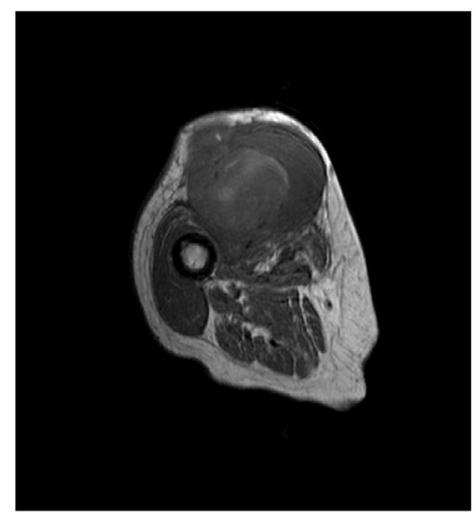

Longitudinal section with contrast

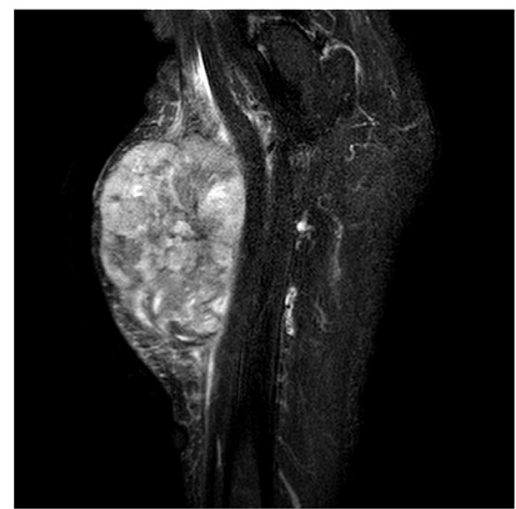

Longitudinal section without contrast

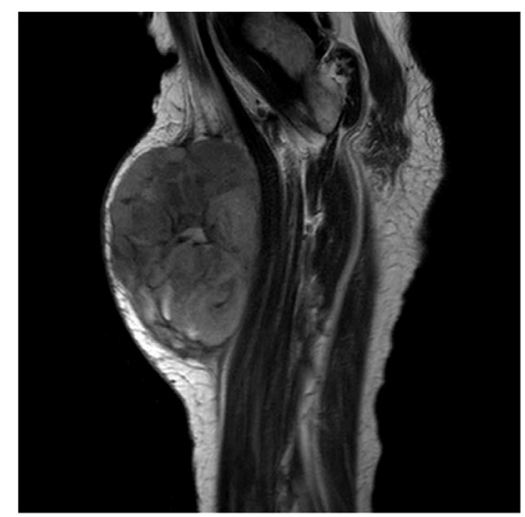

Figure 1. MRI scan: An 80-year-old woman with subcutaneous soft tissue tumor in the lower extremities with relation to both tendons, skin and vital blood vessels. Primary differential diagnosis was sarcoma. A biopsy was performed. Histopathological examinations raised doubt regarding the diagnosis; was it malignant melanoma or clear cell sarcoma. Subsequently verified as a metastasis of malignant melanoma.

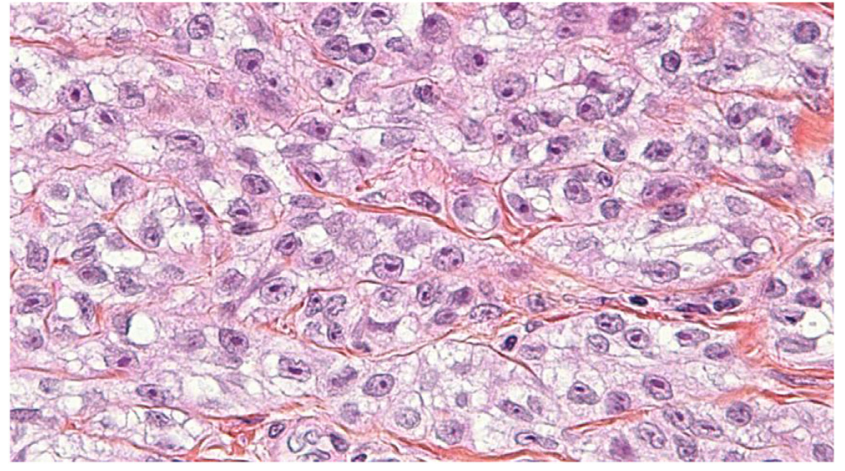

Figure 2. CCS, hematoxylin-eosin stain: There are irregular nests of cells separated by fibrous septa. Cells are fusiform and not pleomorphic. They have regular vesicular nuclei and prominent nucleoli with moderate to abundant eosinophilic or clear cytoplasm.

conventional ultrasound and computed tomography (CT) may be utilized as supplements and primary investigation in the few cases of contraindications to the MRI or because these modalities are more widely available.

When diagnosing CCS, it is important to consider obtaining the best possible preoperative imaging simultaneously, as surgery is the primary and possible curative treatment and further delays in terms of planning radical surgery, may render the prognosis even worse. As such the MRI produces a clear image of the tumor, and its relations to local structures e.g. nerves and major blood vessels, which may result in reduced per operative morbidity due to better planning (Fig. 1).

As CCS typically disseminate by haematogenous spread to the lungs or via the lymphatic system, Positron emission tomography-computed tomography (PET-CT) scan may be used to evaluate patients' status in terms of radical surgery.

\section{Pathological examination}

The final diagnosis of CCS is pathological. Macroscopically the CCS appears to be well-defined solid tumors, with mat grey color that frequently infiltrate tendons and aponeuroses. Microscopically findings are small compact nests with uniform neoplastic cells divided into variable sized clusters by fibrous septa along the tendons and aponeuroses. ${ }^{2,6}$ The tumor cells typically appear with a clear eosinophilic cytoplasm and a basophilic nucleolus (Fig. 2). The eosinophilic cytoplasm is due to the accumulation of glycogen, which can be visualized using periodic (PAS) stain method. The tumor cells show none or a minimal rate of mitosis in concordance with the slow growth rate of CCS. Scattered giant cells with 10-16 nucleoli and scattered necrosis may also be identified in CCS. ${ }^{2}$

Immunohistochemical studies of CCS show great similarity to the characteristics of malignant melanoma, including a strong expression of S-100, HMB-45, Melan-A and microphthalmia-associated transcription factor (MITF) in $81-97 \%$ of the cases. ${ }^{2,19}$ Clear cell sarcoma is associated the chromosomal fusion of the Ewing's Sarcoma oncogene (EWS) and the cellular transcription factor (ATF1) into EWS/ATF1 oncogene. ${ }^{20}$ This chromosomal fusion due to $(t[12 ; 22][q 13 ; q 12])$ can be detected with cytogenetic investigations, e.g. polymerase chain reaction (PCR) and fluorescence in situ hybridization (FISH) in $90 \%$ of the cases. ${ }^{20}$ In addition, to the before mentioned translocation, a fusion between Ewing Sarcoma Breakpoint Region 1(EWSR1) and Camp Responsive Element Binding Protein 1 (CREB1) is also known to give rise to CCS. ${ }^{21}$ Studies have shown that EWSR1/ATFI fusion protein is able to bind and activate melanocyte specific MITF via SRY-related HMG-box 10 (SOX10) transcription factor, which results in the expression of the melanocytic phenotype, thus the malignant melanoma is a differential diagnosis. $^{22,23}$ 


\section{Fédération Nationales des Centres de Lutte Contre le cancer; High Power Field}

\begin{tabular}{|c|c|}
\hline Tumor differentiation & Definition \\
\hline Score 1 & Sarcomas that closely resemble normal adult mesenchymal tissues \\
\hline Score 2 & Sarcomas for which histologic typing is certain \\
\hline Score 3 & Embryonal and undifferentiated sarcomas, synovial sarcoma and sarcomas of uncertain differentiation \\
\hline \multicolumn{2}{|l|}{ Mitotic count } \\
\hline Score 1 & $0-9$ mitosis/ $10 \mathrm{hpf}$ \\
\hline Score 2 & $10-19$ mitosis/ $10 \mathrm{hpf}$ \\
\hline Score 3 & $20 \geq$ mitosis/ 10 hpf \\
\hline \multicolumn{2}{|l|}{ Tumor necrosis } \\
\hline Score 0 & No necrosis \\
\hline Score 1 & $<50 \%$ tumor necrosis \\
\hline Score 2 & $\geq 50 \%$ tumor necrosis \\
\hline Histologic grade & Tumor differentiation + mitotic count + tumor necrosis \\
\hline Grade 1 (Low grade) & Total score: 2 or 3 \\
\hline Grade 2 (Intermediate grade) & Total score: 4 or 5 \\
\hline Grade 3 (High grade) & $<50 \%$ tumor necrosis \\
\hline
\end{tabular}

Figure 3. FNCLCC grading.

\section{Differential diagnosis}

The diagnosis of CCS may be difficult and differential diagnosis are plenty and include melanoma, epithelioid malignant peripheral nerve sheath tumor, cellular blue naevus, melanotic schwannoma, paraganglioma-like dermal melanocytic tumor, synovial sarcoma (monophasic type), paraganglioma, epithelioid sarcoma and carcinomas. ${ }^{24}$ In comparison to melanoma although clinically distinctive, the initial pathological examination may be ambiguous, hence bear in mind that CCS is more deeply located, in close relation to tendons or aponeuroses and with no epidermal involvement. In contrast, a primary cutaneous nodular melanoma is usually situated in the dermis and tends to be more pleomorphic and enlarging more rapidly than CCS. Remember that melanoma metastasis can be found in deeper tissue (Fig. 1). Melanotic schwannoma are rare and most often occurs in patients with Carney syndrome, together with spotty skin and myxomas. These schwannomas are situated in paraspinal or nerve plexus-related locations and frequently contains calcifications and very abundant melanin pigment. $^{24}$

Malignant peripheral nerve sheath tumor is, as indicated in the name, associated with a large peripheral nerve or neurofibromatosis and is histologically characterized by significant myxoid stroma (i.e. pale to light basophilic stroma) in haematoxylin and eosin stains, hyperchromatic nuclei, brisk mitotic activity, negativity for HMB-45, melanocytic markers and cytogenetic abnormalities. ${ }^{3}$ In contrast to CCS, paraganglioma-like dermal melanocytic tumor is a tumor of the extremities of only females, which presents as a dermal nodule with distinct histological appearance, large epithelioid cells with distinct large nuclei, prominent nucleoli and absence of genomic EWS rearrangement.

\section{Treatment}

Margin-free surgery is the primary treatment of CCS. ${ }^{8,9,18,25}$ Recommendations are surgical excision with a sufficient amount of surrounding healthy tissue. Most authors recommend a margin of $1 \mathrm{~cm}$ or more, although this is a matter of ongoing debate. ${ }^{8,9,15}$ Adjuvant treatment as discussed below is recommended in cases, where it is not possible to meet the recommendations of margins. ${ }^{26}$ Amputation following CCS is rarely used in contemporary medicine. ${ }^{15}$ One third of patients if undergoing sentinel node procedure or subsequently lymph node dissection present with lymphatic metastasis and these procedures are discussed in regards of locoregional disease control. ${ }^{8}$ The procedures seem to predict recurrent disease as well as survival but are not yet widely implemented.

\section{Radiation therapy}

Adjuvant radiation therapy can be used as a postoperative adjuvant treatment of CCS. ${ }^{27}$ It is commonly used in delicate situations, where the recommended margin of $1 \mathrm{~cm}$ of healthy tissue cannot be achieved and additional surgery is not feasible or refused by the patient. ${ }^{18,28,29}$

It is unclear whether adjuvant radiotherapy has a beneficial effect on survival. ${ }^{27}$ It is recommended in cases of high grade (grade 2-3) and large $(>5 \mathrm{~cm})$ soft tissue sarcomas (level IIB). ${ }^{30}$ Grading are enumerated in Fig. 3.

Clear cell sarcoma, however, typically has low mitotic activity and necrosis is only seen occasionally in these tumors, classifying most CCSs as grade 1 tumors. ${ }^{29}$ In cases with negative surgical margins, a radiation dosis of $50 \mathrm{~Gy}$ is recommended in fractions of 1.8-2.0 Gy. If positive margins are present a postoperative radiation regime of $60-66 \mathrm{~Gy}$ is recommended. ${ }^{29}$

\section{Chemotherapy}

If complete excision with sufficient margin is achieved, adjuvant treatments are not necessary. ${ }^{26}$ Chemotherapy is indicated in patients with metastatic disease but the efficiency still remains unclear, although it is being used as palliative treatment. ${ }^{25,26,31}$ Hence, in a retrospective study of 24 patients, investigating the response rate of 
metastatic CCS disease and the treatment with chemotherapy the authors found that treatment with anthracycline based chemotherapy was the only therapy with partial effect on metastatic CCS, as the response rate was $4 \% .{ }^{13}$ Both cisplatin and ifosamide based protocols only managed stable disease in two patients with a progression free period of 11 weeks. ${ }^{13}$ Similar findings, in another study in which advanced CCS disease was studied in 11 patients with a combination of doxorubicin, dacarbazine and ifosfamide, as only two patients achieved partial response or stable disease and it lasted less than six months. ${ }^{32}$

\section{Postoperative control}

An extensive follow-up program is advised and instituted in most countries, due to the high risk of metastases and relapse. The purpose is to exclude recurrence and metastases, in addition to address occupational health issues, practical needs, and to support self-care and negate long-term sequelae of the treatment.

The following is recommended. Histopathological low grade tumors (grade 1) are to be followed once every three months the first year, once every four months for two-three years and hereafter twice a year until the fifth postoperative year. ${ }^{33}$ Clinical monitoring is especially important when it comes to local recurrence and clinical examination should be supplemented with MRI on any suspicion. ${ }^{34}$ Patients with high grade tumors (grades 2-3) are recommended to follow a rigid scheme of controls every three months for the first three years and then twice a year through the fifth year. ${ }^{33}$ Even with a negative clinical examination, it is still recommended to have regular MRI of previous tumor site twice a year. The exact role of PET-CT and chest x-ray is not fully established but often used on suspicion in order to exclude metastases. ${ }^{35}$ Individualized control programs can occur in up to ten years postoperatively. Patients that have received chemotherapy should also be examined biochemically with liver, kidney, and heart function parameters because of the risk of side-effects.

\section{Prognosis}

The prognosis of CCS is poor because of the tendency to metastasize at an early disease-state and due to the rate of recurrence. ${ }^{8,15,18}$ Local recurrence rate may be as high as $84 \%$ and $30 \%$ of patients have metastases at the time of diagnosis. ${ }^{7,15,36}$ Negatively associated prognostic factors are necrosis, the number of mitosis, resection margin, anatomic location and tumor size. ${ }^{11,15,26}$ Tumor size, at the time of surgery, seems to be the key factor and is most strongly correlated to survival. Tumors larger than $5 \mathrm{~cm}$ are proven to recur more often because of micro metastasis. ${ }^{11,19,25}$

\section{Conclusion}

CCS is a rare malignant disease with very low incidence of the soft tissue with numerous differential diagnoses. The incidence is very low and the prognosis is poor. Primary treatment is radical surgical extirpation and an extensive postoperative follow-up program. The role of adjuvant therapy i.e. radiation and chemotherapy remains to be elucidated and larger prospective studies are required to establish the optimal treatment.

\section{References}

1. Antonescu CR, Tschernyavsky SJ, Woodruff JM, et al. Molecular diagnosis of clear cell sarcoma: detection of EWS-ATF1 and MITF-M transcripts and histopathological and ultrastructural analysis of 12 cases. J Mol Diagn. 2002;4:44-52.
2. Kindblom LG, Lodding P, Angervall L. Clear-cell sarcoma of tendons and aponeuroses. An immunohistochemical and electron microscopic analysis indicating neural crest origin. Virchows Arch A Pathol Anat Histopathol. 1983;401:109-128.

3. Dim DC, Cooley LD, Miranda RN. Clear cell sarcoma of tendons and aponeuroses: a review. Arch Pathol Lab Med. 2007;131:152-156.

4. García de Marcos JA, del Castillo-Pardo de Vera JL, Poblet E, et al. Clear cell sarcoma of the temporal region: case report, review of the literature, and genetic analysis. $J$ Oral Maxillofac Surg. 2009;67:910-914.

5. Shmookler B, Bickels J, Jelinek J, et al. Bone and soft-tissue Sarcomas : epidemiology, radiology, pathology and fundamentals of surgical treatment. Musculoskelet Cancer Surg Treat Sarcomas Allied Dis. 2001:3-36.

6. ENZINGER FM. Clear-Cell sarcoma of tendons and aponeuroses. An analysis of 21 cases. Cancer. 1965;18:1163-1174.

7. Chung EB, Enzinger FM. Malignant melanoma of soft parts. A reassessment of clear cell sarcoma. Am J Surg Pathol. 1983;7:405-413.

8. van Akkooi a CJ, Verhoef C, van Geel a N, et al. Sentinel node biopsy for clear cell sarcoma. Eur J Surg Oncol. 2006;32:996-999.

9. Isoda H, Kuroda M, Saitoh M, et al. MR findings of clear cell sarcoma: two case reports. Clin Imaging; 27: 229-232.

10. Kuiper DR, Hoekstra HJ, Veth RPH, et al. The management of clear cell sarcoma. Eur J Surg Oncol. 2003;29:568-570.

11. Deenik W, Mooi WJ, Rutgers EJ, et al. Clear cell sarcoma (malignant melanoma) of soft parts: a clinicopathologic study of 30 cases. Cancer. 1999;86:969-975.

12. Eckardt JJ, Pritchard DJ, Soule EH. Clear cell sarcoma. A clinicopathologic study of 27 cases. Cancer. 1983;52:1482-1488.

13. Jones RL, Constantinidou A, Thway K, et al. Chemotherapy in clear cell sarcoma. Med Oncol. 2011;28:859-863.

14. Malchau SS, Hayden J, Hornicek F, et al. Clear cell sarcoma of soft tissues. J Surg Oncol. 2007;95:519-522.

15. Lucas DR, Nascimento AG, Sim FH. Clear cell sarcoma of soft tissues. Mayo Clinic experience with 35 cases. Am J Surg Pathol. 1992;16:1197-1204.

16. Gielen JLMA, De Schepper AM, Vanhoenacker F, et al. Accuracy of MRI in characterization of soft tissue tumors and tumor-like lesions. A prospective study in 548 patients. Eur Radiol. 2004:14:2320-2330.

17. Kransdorf MJ MM. No Title. Imaging Soft Tissue Tumors; 2.

18. Kuiper DR, Hoekstra HJ, Veth RPH, et al. The management of clear cell sarcoma. Eur J Surg Oncol 2003:29:568-570.

19. Hisaoka M, Ishida T, Kuo T-T, et al. Clear cell sarcoma of soft tissue: a clinicopathologic, immunohistochemical, and molecular analysis of 33 cases. Am J Surg Pathol. 2008;32:452-460

20. Wang W-L, Mayordomo E, Zhang W, et al. Detection and characterization of EWSR1 ATF1 and EWSR1/CREB1 chimeric transcripts in clear cell sarcoma (melanoma of soft parts). Mod Pathol. 2009;22:1201-1209.

21. Liu C, Ren Y, Li X, et al. Absence of 19 known hotspot oncogenic mutations in soft tissue clear cell sarcoma: two cases report with review of the literature. Int J Clin Exp Pathol. 2014;7:5242-5249.

22. Davis IJ, Kim JJ, Ozsolak F, et al. Oncogenic MITF dysregulation in clear cell sarcoma: defining the MiT family of human cancers. Cancer Cell. 2006;9:473-484.

23. Li KKC, Goodall J, Goding CR, et al. The melanocyte inducing factor MITF is stably expressed in cell lines from human clear cell sarcoma. Br J Cancer. 2003;89:1072-1078

24. Kosemehmetoglu K, Folpe AL. Clear cell sarcoma of tendons and aponeuroses, and osteoclast-rich tumour of the gastrointestinal tract with features resembling clear cell sarcoma of soft parts: a review and update. J Clin Pathol. 2010;63:416-423.

25. Kawai A, Hosono A, Nakayama R, et al. Clear cell sarcoma of tendons and aponeuroses: a study of 75 patients. Cancer. 2007;109:109-116.

26. Ferrari A, Casanova M, Bisogno G, et al. Clear cell sarcoma of tendons and aponeuroses in pediatric patients: a report from the Italian and German Soft Tissue Sarcoma Cooperative Group. Cancer. 2002;94:3269-3276.

27. Al-Absi E, Farrokhyar F, Sharma R, et al. A systematic review and meta-analysis of oncologic outcomes of pre- versus postoperative radiation in localized resectable soft-tissue sarcoma. Ann Surg Oncol. 2010;17:1367-1374.

28. Deenik W, Mooi WJ, Rutgers EJ, et al. Clear cell sarcoma (malignant melanoma) of soft parts: a clinicopathologic study of 30 cases. Cancer. 1999;86:969-975.

29. Enneking WF, Spanier SS, Goodman MA. A system for the surgical staging of musculoskeletal sarcoma. 1980. Clin Orthop Relat Res. 2003:4-18.

30. ESMO/European Sarcoma Network Working Group. Soft tissue and visceral sarcomas: ESMO Clinical Practice Guidelines for diagnosis, treatment and follow-up. Ann Oncol Off J Eur Soc Med Oncol. 2014;25(Suppl 3):iii102-i112.

31. Steger GG, Wrba F, Mader R, et al. Complete remission of metastasised clear cell sarcoma of tendons and aponeuroses. Eur J Cancer. 1991;27:254-256.

32. Stacchiotti S, Palassini T, Negri M et al. Clear Cell Sarcoma (CCR): Clinical Behavior and Response to Chemotherapy. Poster Present; American S.

33. Rutkowski P, Ługowska I. Follow-up in soft tissue sarcomas. Memo - Mag Eur Med Oncol. 2014;7:92-96.

34. Hovedanbefalinger DS. Bilag Til Kræftplan II.

35. National Guidelines for Treatment of Sarcoma, Norway.

36. Marquès $\mathrm{B}$, Terrier $\mathrm{P}$, Voigt JJ, et al. Clear cell soft tissue sarcoma. Clinical, histopathological and prognostic study of 36 cases. Ann Pathol. 2000;20:298-303. 